JASC 12-2-4

\title{
Automatic blood pressure measurement device using oscillometric method and Korotkoff sounds
}

\author{
Ran Wei*, Young Chul Lim**, Jae Joong Im**† \\ *School of Electronics and Information Engineering, Tianjin Polytechnic University, Tianjin, China \\ **Division of Electronic Engineering, Chonbuk National University, Jeonju, Korea \\ †Division of Electronic Engineering, Chonbuk National University, Jeonju, Korea
}

\begin{abstract}
The oscillometric method and Korotkoff sound method are the most common ways to measure the blood pressure. A new automatic blood pressure measurement device, which uses both oscillometric method and Korotkoff method, was developed. A pressure sensor was used to obtain cuff pressure and oscillation signal, and a microphone was used to detect Korotkoff sounds. Forty-five measurements from fifteen subjects were used for analysis. Correlation coefficients between the traditional auscultatory method and Korotkoff sound method were 0.9820 and 0.9721 for the systolic and diastolic blood pressure values, respectively. Standard deviations of differences for the systolic and diastolic blood pressure values were 1.3019 and 1.4495 , respectively. Correspondingly, correlation coefficients between the traditional auscultatory method and oscillometric method using newly developed algorithm were 0.9651 and 0.9136 for the systolic and diastolic blood pressure values, with the standard deviations of 1.42 and 1.73 , respectively. The results showed that the newly developed algorithm for oscillometirc method provide accurate blood pressure values, moreover, Korotkoff sound method using microphone provides even higher accuracy. Therefore, a new automatic device which utilizes both oscillometric method and Korotkoff sound method would provide the accurate and reliable blood pressure values.
\end{abstract}

Key words: blood pressure, oscillometric, korotkoff sounds, PVDF

\section{INTRODUCTION}

Blood pressure, which indicates whether the blood is normally circulating to the body tissues, is a powerful, consistent, and independent risk factor for cardiovascular disease $^{[1][2][3]}$. The values of blood pressure help the physicians determine the functional integrity of the cardiovascular system and used for broad clinical applications. Basically, both direct (invasive) and indirect (noninvasive) techniques are used to measure blood pressure.

Direct measurement of blood pressure may be done by inserting a sterile, saline-filled catheter into the blood vessel. The pressure at the tip of the catheter is then transmitted to a

Manuscript received : Sept. 09, 2012 / revised : Nov. 1, 2012

${ }^{\dagger}$ Corresponding Author: jjim@jbnu.ac.kr

Tel: +82-63-270-2409, Fax: +82-63-270-2394,

Division of Electronic Engineering, Chonbuk National University, Jeonju, Korea pressure transducer. This method is used only during operation or in the intensive care unit, and imposes many risks and problems during process, especially for nondestructive sterilization. Indirect measurement of blood pressure is an attempt to measure intra-arterial pressures noninvasively. Various attempts are used to measure the blood pressure indirectly such as auscultatory, oscillometry, ultrasound, and tonometry methods. Brachial artery is the most common site for the indirect measurement because of its convenience, and the auscultatory and oscillometric methods are the most common indirect methods. The auscultatory method uses a sphygmomanometer, which consists of an inflatable cuff for occlusion of the blood vessel, a rubber bulb for inflation of the cuff, and either a mercury or an aneroid manometer for pressure detection. It auscultates the sounds using a stethoscope or microphone to find the starting and ending point of the Korotkoff sounds as the cuff pressure is decreased ${ }^{[4][5]}$. Comparatively, the oscillometric method detects the oscillations in the cuff, and 
calculates the values of blood pressure by establishing an algorithm.

All of these methods have their advantages and disadvantages ${ }^{[6][7][8]}$. The invasive methods are more accurate, but complex and inconvenient. Correspondingly, the noninvasive devices are safer and easier to use, leads the application more extended. Comparatively, the oscillometric method detects the pressure oscillations in the cuff. This method is based on the registration of the amplitudes changes, which corresponds with the oscillation of the pulse waves. The point of maximal oscillation corresponds to the mean intra-arterial pressure when the oscillations of pressure in a sphygmomanometer cuff are recorded during gradual deflation. It is observed that systolic pressure was encountered when the amplitude of cuff pressure oscillation reaches about $57-45 \%$ of the maximum amplitude, while diastolic pressure was encountered when the amplitude of the pressure oscillation becomes about $75-86 \%$ of the maximum amplitude $\mathrm{e}^{[9][10]}$. However, auscultatory method is difficult to be used in noisy environment, and the bias exists among the observers. On the other hand, the oscillometric method can not be applied to the carotid or femoral, and there are no standard algorithms exist leading to large variance in blood pressures values from different devices.

It calls for the development of a new device which increases the accuracy of the automatic blood pressure measurement device ${ }^{[11][12]}$. The objective of this study was to develop a new automatic blood pressure measurement device which utilizes both oscillometric method and Korotkoff sound method. The device includes a pressure sensor for obtaining cuff pressure and oscillation signal, and a microphone for detecting Korotkoff sounds. Hardware circuits are established for the processing of analog signals and a new algorithm for determining blood pressure values is suggested. The results of the study will be used for the development of a new device for accurate blood pressure measurement. The new system could also be used for the monitoring of 24-hour ambulatory blood pressure monitoring devices. Moreover, the resulting algorithm for the oscillometric method could provide the basic concepts for the development of a noninvasive continuous cuffless blood pressure monitoring device.

\section{MethodS}

This study was performed to develop an accurate automatic blood pressure measurement device which calculates blood pressure values based on the oscillometric method and Korotkoff sound method. The device includes a pressure sensor, microphone, hardware circuits, and new algorithm for determining blood pressure values based on relationship between oscillometric method and Korotkoff sound method.

\subsection{Experimental design}

A microphone using PVDF(polyvinyledene fluoride) film and a pressure sensor (FPN-05PG, Fujikura Ltd., Japan) was used to obtain Korotkoff sounds and absolute cuff pressure, respectively. The pressure sensor was also used for acquiring variation of pressure oscillations as the cuff pressure is decreased. The hardware was composed of high-pass, low-pass, $60 \mathrm{~Hz}$ notch filters and amplifiers, and data obtained from $\mathrm{A} / \mathrm{D}$ conversion were analyzed to calculate blood pressure values. Finally, the results were evaluated to validate the accuracy.

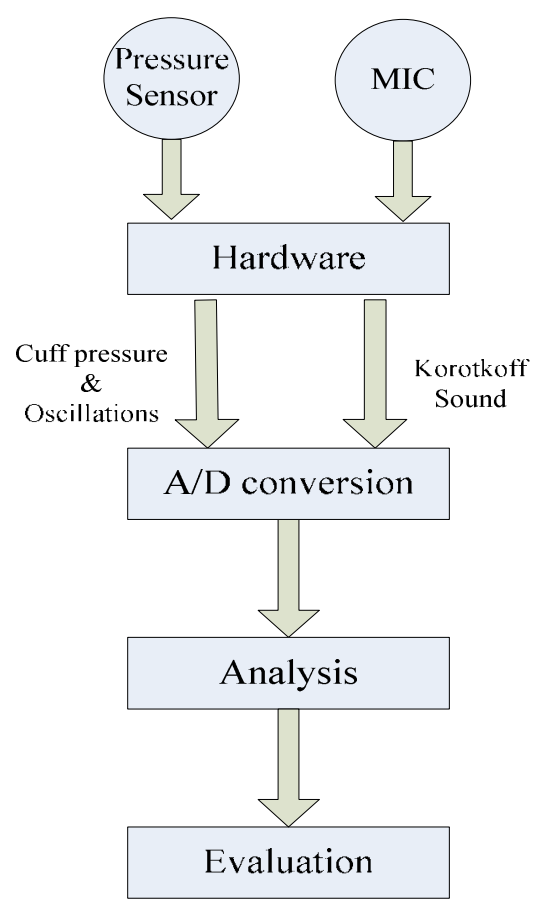

Fig. 1. Overall experimental design for the study

\subsection{Sensor and hardware design}

Microphone was used for detecting Korotkoff sounds. The contact microphone uses sensitive PVDF film combined with a low-noise electronic preamplifier in order to minimize external acoustic noise while offering extremely high sensitivity to vibration applied to the central rubber pad. The microphone is shown in Figure 2. The $30 \mathrm{~Hz}$ high-pass filter and $300 \mathrm{~Hz}$ low-pass filter were used to acquire clear Korotkoff sounds, and then the amplifier was used to amplify the Korotkoff signals to the appropriate levels. 


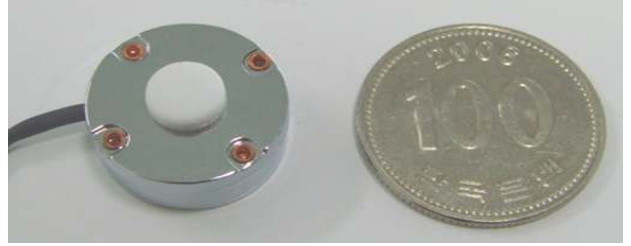

Figure 2. The contact microphone usgin PVDF film

Pressure sensor was used to obtain absolute cuff pressure and cuff pressure oscillations. Figure 3 shows the diagram of pressure sensor configuration. The measurable range of the pressure sensor (FPN-05PG, Fujikura Ltd., Japan) was from $-34.47 \mathrm{KPa}$ to $34.47 \mathrm{KPa}$ and the full scale span voltage is from $40 \mathrm{mV}$ to $130 \mathrm{mV}$.

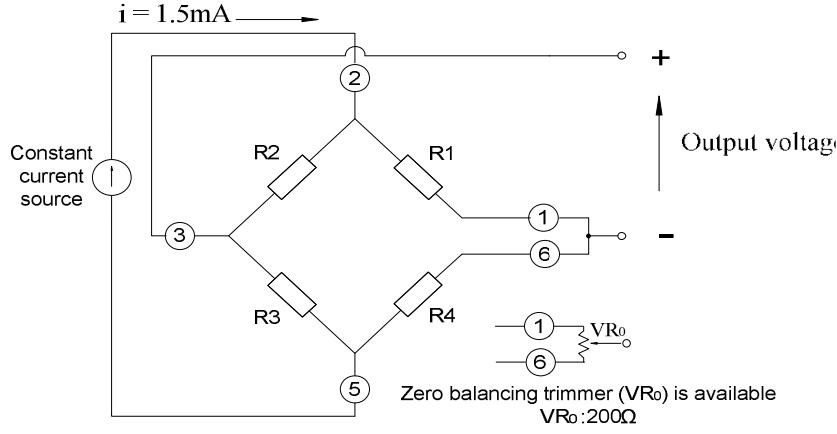

Fig. 3. Connection diagram of the pressure sensor

According to the measurable pressure range and the full scale span voltage of the FPN-05PG pressure sensor, slope of the pressure change was calculated as;

Slope $=\frac{V_{\max }}{P_{\max }}=\frac{130 \mathrm{mV}}{34.47 \mathrm{KPa}} \times \frac{101.325 \mathrm{KPa}}{760 \mathrm{mmHg}}=0.503 \mathrm{mv} / \mathrm{mmHg}$

The pressure in the cuff in the unit of $\mathrm{mmHg}$ was calculated as:

$\operatorname{Pressure}{ }_{m m H g}=\frac{V_{\text {output }}}{V_{\text {gain }}} \times \frac{1}{\text { slope }}$

where the values of Vgain was calculated by the amplifier. The cuff pressure oscillations were superimposed on cuff pressure. Therefore, the $0.3 \mathrm{~Hz}$ high-pass filter and $30 \mathrm{~Hz}$ low-pass filter were used to separate the cuff oscillations from the pressure sensor.

\subsection{Data acquisition}

Fifteen healthy subjects, 8 males and 7 females, ranging in systolic pressure from 94 to $128 \mathrm{mmHg}$, and in diastolic pressure from 66 to $86 \mathrm{mmHg}$, were participated for the study. Preliminary steps of obtaining data consist of placing the subjects in the comfortable and recumbent position for more than 5 minutes, in an attempt to allay fear and anxiety. Then, blood pressure was measured using a standard $13-\mathrm{cm}$ wide cuff on the left arm at the heart level to obtain reference values of systolic and diastolic blood pressure.

After the standard measure of blood pressure values from the subjects, 45 measurements (three measurements from each subject) from the 15 subjects were recorded by the data acquisition system, MP-100 (BIOPAC systems, USA). Cuff was inflated to above $200 \mathrm{mmHg}$, then deflated at the rate of about 3 or $4 \mathrm{mmHg}$ per heart beat. Three signals, Korotkoff sounds, oscillations and absolute cuff pressure, were acquired at the sampling rate of 200 samples per second for the duration of 90 seconds. After each trial of data collection, data were stored for further processing and analysis.

\subsection{Data analysis}

In order to establish a new algorithm for the calculation of blood pressure values out of oscillometirc and korotkoff sound methods, relationship among three variables, absolute pressure values, pressure oscillation, and Korotkoff sounds, should be determined. Once the accurate systolic and diastolic blood pressures were obtained from the Korotkoff sounds, corresponding pressure oscillation signal were analyzed.

According to the measurable pressure range and the full scale span voltage of the FPN-05PG pressure sensor, slope of the pressure change was calculated as in equation (3).

Slope $=\frac{V_{\max }}{P_{\max }}=\frac{130 \mathrm{mV}}{34.47 \mathrm{KPa}} \times \frac{101.325 \mathrm{KPa}}{760 \mathrm{mmHg}}=0.502812 \mathrm{mv} / \mathrm{mmHg}$

The pressure in the cuff and the values of $\mathrm{V}_{\text {gain }}$ was set at 50. Also, the pressure in the cuff in the unit of $\mathrm{mmHg}$ was calculated as in equation (5).

$$
\begin{aligned}
& \text { Pr } \text { essure }_{\text {mm Hg }}=\frac{V_{\text {ouput }}}{V_{\text {gain }}} \times \frac{1}{\text { slope }}=\frac{V_{\text {output }}}{V_{\text {gain }}} \times 1.988815 \times 10^{3} \mathrm{~mm} \mathrm{Hg} / \mathrm{V} \\
& \text { Pr } \text { essure }_{m m H g}=\frac{V_{\text {output }}}{V_{\text {gain }}} \times \frac{1}{\text { slope }}=V_{\text {output }} \times 39.776 \mathrm{~mm} \mathrm{Hg} / \mathrm{V}
\end{aligned}
$$

Absolute cuff pressure, Korotkoff sounds, and cuff pressure oscillations were acquired simultaneously as can be seen from Figure 4. In order to develop a new algorithm for oscillometric method, five parameters were extracted from the waveforms, (1) the cuff pressure at the Korotkoff systolic pressure $\left(\mathrm{K}_{\mathrm{S}}\right),(2)$ the cuff pressure at the Korotkoff diastolic pressure $\left(\mathrm{K}_{\mathrm{D}}\right)$, (3) the maximal amplitude at the pressure oscillations $\left(\mathrm{O}_{\mathrm{M}}\right)$, (4) the amplitude $\left(\mathrm{O}_{\mathrm{S}}\right)$ at the Korotkoff systolic pressure, and (5) the amplitude at the Korotkoff diastolic pressure $\left(\mathrm{O}_{D}\right)$. Finally, the ratio $\mathrm{O}_{S} / \mathrm{O}_{M}$ and $\mathrm{O}_{\mathrm{D}} / \mathrm{O}_{\mathrm{M}}$ were calculated to identify the parameters of the oscillometric algorithms. 


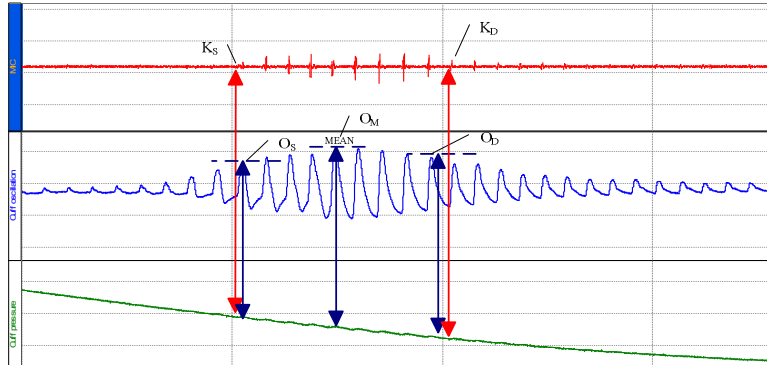

Fig. 4. Graph and illustration of the Korotkoff sound, cuff oscillation, and cuff pressure

\section{RESULTS}

Since the traditional auscultatory method other than invasive method could be the only accurate measurement for the blood pressure. Auscultatory method using mercury manometer, cuff and stethoscope was used as a reference for evaluating the results calculated based on the new method

The mean differences and standard deviations between the auscultatory and oscillometric methods are shown in Table 1. As can be seen from the table, the mean systolic differences are $1.80 \mathrm{mmHg}$ with the standard deviations of 1.42. Mean diastolic differences are $2.09 \mathrm{mmHg}$ with the standard deviations of 1.73 . The mean differences and standard deviations between the auscultatory and Korotkoff sound methods are shown in Table 2. As can be seen from the table, the mean systolic differences are $1.2 \mathrm{mmHg}$ with the standard deviations of 1.65. Mean diastolic differences are $0.73 \mathrm{mmHg}$ with the standard deviations of 1.15 .

Table 1. The mean differences and standard deviations between the auscultatory and oscillometric methods

\begin{tabular}{lll}
\hline \hline & $\begin{array}{l}\text { Mean Differences } \\
(\mathrm{mmHg})\end{array}$ & $\begin{array}{l}\text { Standard } \\
\text { deviation }\end{array}$ \\
\hline $\begin{array}{l}\text { Systolic Pressure } \\
\text { Diastolic }\end{array}$ & 1.80 & 1.42 \\
Pressure & 2.09 & 1.73 \\
\hline \hline
\end{tabular}

Table 2. The mean differences and standard deviations between the auscultatory and Korotkoff sound methods by using two algorithms

\begin{tabular}{lll}
\hline \hline & $\begin{array}{l}\text { Mean Differences } \\
(\mathrm{mmHg})\end{array}$ & $\begin{array}{l}\text { Standard } \\
\text { deviation }\end{array}$ \\
\hline $\begin{array}{l}\text { Systolic Pressure } \\
\begin{array}{l}\text { Diastolic } \\
\text { Pressure }\end{array}\end{array}$ & 1.20 & 1.65 \\
\hline \hline
\end{tabular}

It has been clearly observed that the oscillometric method provides less accurate results than the Korotkoff sound method. Since the principle of the auscultatory method was based on hearing Korotkoff sound, it is less prone to provide the false readings. However, there exist variability caused by observer bias, using different devices, and use of microphone instead of relying on human hearing abilities. On the other hand, there exist some disadvantages for the Korotkoff method. For example, the placement of the microphone and the rate of deflating affect the accurate measurement of the blood pressure.

Figure 5 presents the correlation of the systolic and diastolic blood pressure values obtained by auscultatory method and calculated by the mean values of Korotkoff method and oscillometric method. Correlation coefficients are 0.9842 and 0.9862 for the systolic and diastolic blood pressure values, respectively. Standard deviations of differences between those two methods are 1.6787 and $1.1461 \mathrm{mmHg}$ for the systolic and diastolic blood pressure values, respectively.

Figure 6 shows the Bland-Altman plot of the differences of systolic and diastolic blood pressure between the values obtained by the auscultatory method and these calculated by the mean values of Korotkoff method and oscillometric method, respectively. The $\mathrm{x}$-axis represents the mean of the device and observer measurements, and the y-axis represents the difference between the device and observer measurements. Mean differences are 1.2 and $0.73 \mathrm{mmHg}$ for the systolic and diastolic blood pressure values, respectively, and standard deviations of differences between those two methods are $1.6528 \mathrm{mmHg}$ and $1.1461 \mathrm{mmHg}$ for the systolic and diastolic blood pressure values, respectively.

By comparing the previous results obtained using Korotkoff sound method and oscillometric method, it provides much higher accurate results when combining both methods. However, it is under the assumption that the values obtained from each method are accurate. That is, if the Korotkoff sounds are failed to be detected, the mean values of the two methods will not improve the accuracy. Therefore, the further research will consider how to combine the two methods to improve the accuracy.

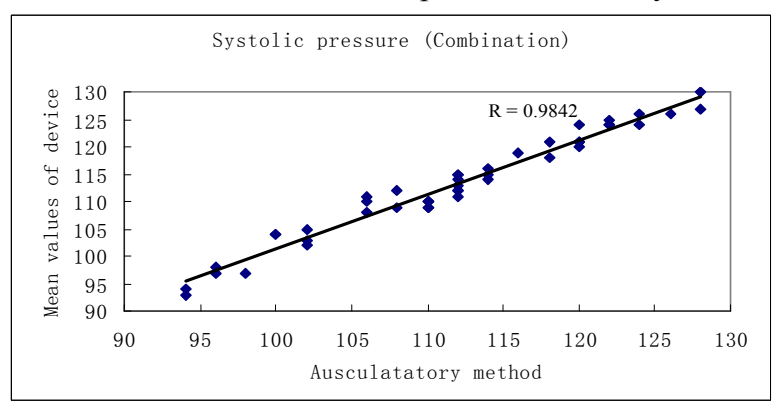

(a) Correlation of systolic pressure values 


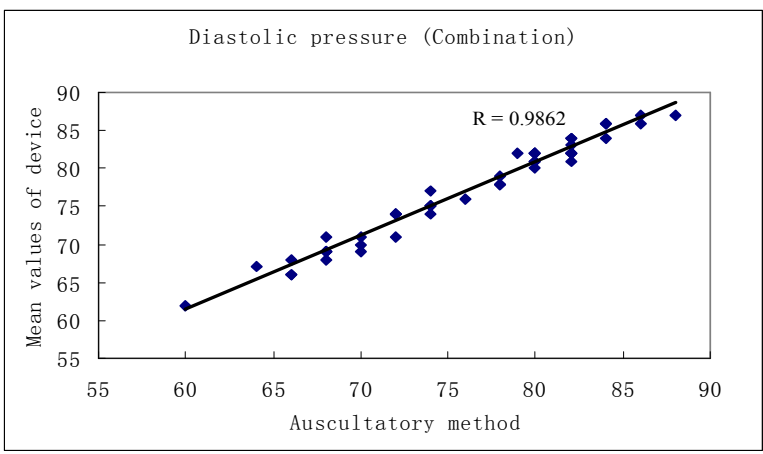

(b) Correlation of diastolic pressure values

Fig. 5. Correlation of the systolic and diastolic blood pressure values obtained between auscultatory method and mean values of Korotkoff method and oscillometric method

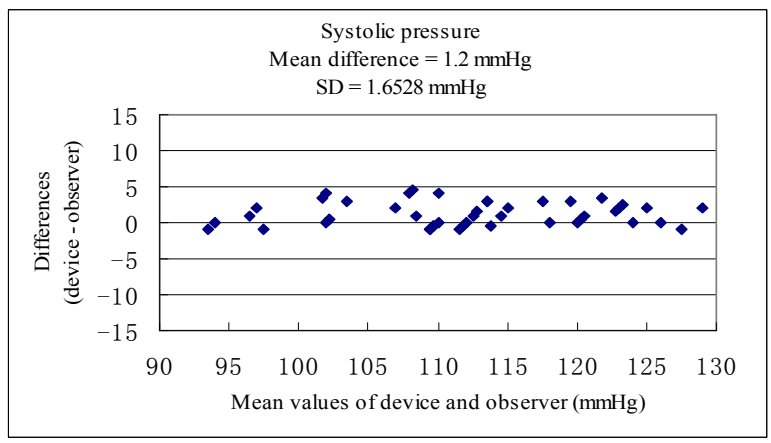

(a) Bland-Altman plot of the systolic pressure values

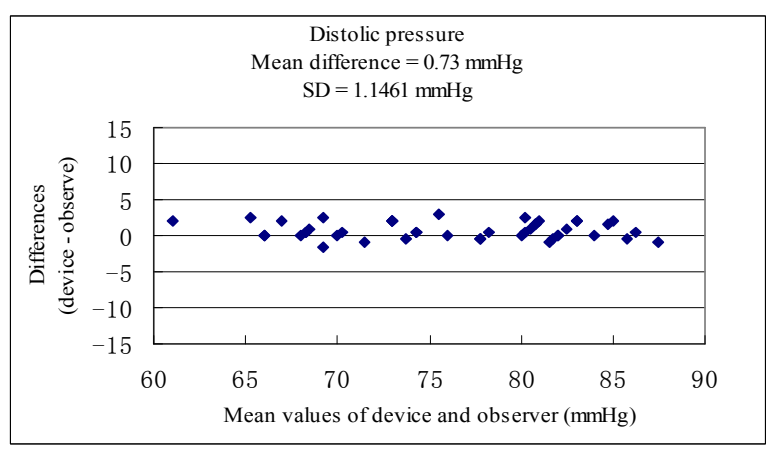

(b) Bland-Altman plot of the diastolic pressure values

Fig. 6. Bland-Altman plot of the differences of systolic blood pressure between the values obtained by the auscultatory method and those calculated by the mean values of Korotkoff method and oscillometric method

\section{CONCLUSIONS}

Among various types of automatic devices for measuring blood pressure, there exist problems of accuracies and reproducibility. Major reasons are the lack of standard algorithm for an oscillometric method which mostly has been used for the current automatic blood pressure measurement devices. It calls for the development of a new device which utilizes improves the accuracy.

This study was designed to utilize both auscultatory method which is based on listening Korotkoff sound and oscillometric method. Also, this study was performed to develop a new algorithm for an oscillometric method and to use such algorithm in addition to the auscultatory method using microphone imbedded inside the cuff. Pressure sensor and microphone were used to design a system, and a new algorithm for an oscillometric method was established.

Conclusions were drawn from the study. First, systolic and diastolic blood pressure values obtained using Korotkoff sound method provides accurate results compare to the traditional auscultatory method. Second, systolic and diastolic blood pressure values obtained using both Korotkoff sounds method and oscillometric method is considered to be more accurate compare to the previous studies. Thirdly, placement of microphone influences the sound quality, therefore, in order to acquire clear Korotkoff sound signals, it is necessary to find the appropriate position for a microphone inside the cuff. Fourth, it is necessary to perform the study with various types of sensors, pressure sensor and microphone. Finally, it is necessary to perform large cohort study, categorized based on various vascular disease and various levels of blood pressure values, for the evaluation of developed algorithm.

This study could be extended for the development of a new automatic blood pressure measurement device based on both auscultatory and oscillometric methods, which could provide accurate and reliable blood pressure values. Once the device is developed, evaluation process could be applied for the international standsard, AAMI (association for the advancement of medical instrumentation) and BHS (british hypertension society). Moreover, the resulting algorithm could be used for the development of a cuffless blood pressure measurement device, which measures the pulse pressure either from brachial artery or from radial artery.

\section{REFERENCES}

[1] C.M. McEniery, I.B. Wilkinson, A.P. Avolio, "Age, Hypertension and Arterial Function.”, Clinical \& Experimental Pharmacology \& Physiology, vol. 34, no. 7, pp. 665-671, July 2007.

[2] S.J. Zieman, V. Melenovsky, D.A. Kass, "Mechanisms, pathophysiology, and therapy of arterial stiffness", Arteriosclerosis, Thrombosis, and Vascular Biology, vol. 25, pp. 932-943, February 2005.

[3] E.K. Thomas, J.S. Robert, S.H. Rebecca, "JNC 7-It's More Than High Blood Pressure", The Journal of the American Medical Association, vol. 289, no. 19, pp. 2573-2575, May 2003. 
[4] S.S. Chio, E.M. Urbina, J. Lapointes, J. Tsai, G.S. Berenson, "Korotkoff sound versus oscillometric cuff sphygmomanometers: comparison between auscultatory and DynaPulse blood pressure measurements", Journal of the American Society of Hypertension, vol. 5, no. 1, pp. 12-20, January-February 2011.

[5] J.S. Williams, S. Brown, P.R. Conlin, "Videos in Clinical Medicine Blood Pressure Measurement", The New England Journal of Medicaine. vol. 360, no. 5, pp. 1-9, January 2009.

[6] R. Raamat, K. Jagomägi, J. Talts, I. Mäger, "Finger beat-to-beat blood pressure responses to successive hand elevations", Medical Engineering \& Physics, vol. 31, no 5, pp.522-527, June 2009.

[7] S. Jones, H. Simpson, H. Ahmed, "A comparison of two methods of blood pressure measurement.", British Journal of Nursing, vol. 15, no. 17, pp. 948-951, October 2006.

[8] J.A. Clark, M.W. Lieh-Lai, A. Sarnaik, T.K. Mattoo, "Discrepancies Between Direct and Indirect Blood Pressure Measurements Using Various Recommendations for Arm Cuff Selection", Pediatrics, Vol. 110, No. 5, pp. 920-923, November 2002.

[9] C.F. Babbs, "Oscillometric measurement of systolic and diastolic blood pressures validated in a physiologic mathematical model", BioMedical Engineering OnLine, vol. 11, no. 1, August 2012.

[10] M. Forouzanfar, H.R. Dajani, V.Z. Groza, M. Bolic, S. Rajan, "Feature-Based Neural Network Approach for Oscillometric Blood Pressure Estimation",IEEE Transactions On Instrumentation and Measurement, vol. 60, no. 8, pp. 2786-2796, August 2011.

[11] M.R. Nelson, S. Quinn, T.M. Winzenberg, F. Howes, L. Shiel, C.M. Reid, "Ankle-Brachial Index determination and peripheral arterial disease diagnosis by an oscillometric blood pressure device in primary care: validation and diagnostic accuracy study.”, BMJ Open, vol. 2, no. 5, October 2012.

[12] Y. Wan, C. Heneghan, R. Stevens, R.J. McManus, A. Ward, R. Perera, M. Thompson, L. Tarassenko, D. Mant. "Determining which automatic digital blood pressure device performs adequately: a systematic review.", Journal of Human Hypertension, vol. 24, no. 7, pp. 431-438, April 2010.

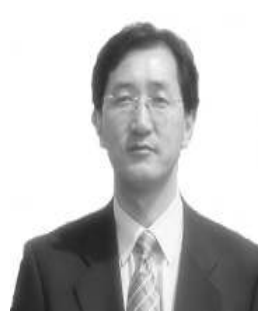

Jae Joong Im (Member)

1983: Department of Electronic Engineering, Konkuk University, Korea (bachelor's)

1988: Department of Biomedical Engineering, Texas A \& M University, USA

(Master)

- 1991: Department of Biomedical Engineering, Texas A \& M University, USA (Ph.D.)

- 1992 - 1998: Department of Biomedical Engineering, Inje University, Korea, Assistant professor

- 1998 - present: Division of Electronic Engineering, Chonbuk National University, Korea, Professor

$<$ Interested in: signal processing, biomedical instrumentation, biosystem simulation>

e-mail: jjim@jbnu.ac.kr

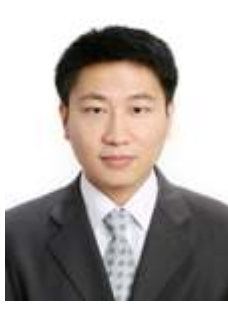

(Master)

- 2012 : Division of Electronic Engineering, Chonbuk National University, Korea (Ph.D.)

- 2012 - present : School of Electronics and Information Engineering, Tianjin Polytechnic University, China, Lecturer $<$ Interested in: signal processing, biomedical instrumentation,> E-mail: weirancn@hotmail.com

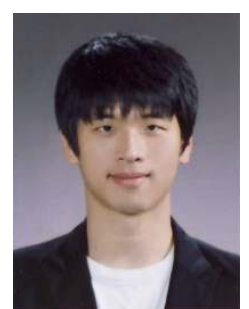

Young Chul Lim

- 2012 : Division of Electronic Engineering, Chonbuk National University, Korea (bachelor's)

- 2012 - present: Division of Electronic Engineering, Chonbuk National University, Korea (Master)

$<$ Interested in: signal processing, biomedical instrumentation> e-mail: 1yc951@nate.com 